cleaning years and incidences of asthma were estimated using Poisson regression analysis. The analyses were repeated in an inception cohort among workers aged 16-20 years at start of follow-up (person years: cleaners $=153,549$; references $=$ 423,506).

Results The risk of asthma was not increased for recent cleaning compared to references (adjusted incidence rate ratio $[$ aIRR $]=1.02$ [95\% confidence interval (CI) 0.99-1.04]). Similar results were seen for recent cleaning in the inception cohort. Cumulated cleaning years (up to 10 years) showed decreased risk of asthma (aIRR $=0.74$ [95\% CI $0.63-0.88$ ] for 10 compared to 1 year of cleaning). However, in the inception cohort (up to 6 years) cumulated cleaning years were associated with increased asthma risk (aIRR $=2.53[95 \%$ CI: 1.38-4.64] for 6 compared to 1 cleaning year).

Conclusion In this study, asthma risk increased with cumulated years of cleaning in the inception cohort. This indicates a strong healthy worker selection and suggests that long-term professional cleaning may be associated with increased risk of asthma. However, in the full population we could not confirm that recent work within cleaning was associated with increased risk of asthma; furthermore, cumulated years of cleaning was inversed associated with asthma.

\section{S-145 USE OF MECHANISTIC EVIDENCE FROM OCCUPATIONAL STUDIES IN CANCER HAZARD IDENTIFICATION: THE EXPERIENCE OF THE IARC MONOGRAPHS PROGRAMME}

${ }^{1}$ Mary Schubauer-Berigan, Kathryn Guyton. 'International Agency for Research on Cancer, France

\subsection{6/OEM-2021-EPI.415}

Over the past 50 years, the Monographs Programme of the International Agency for Research on Cancer (IARC) has evaluated the potential carcinogenic hazard of more than 1000 agents. Through 129 volumes, 121 agents have been classified as 'carcinogenic' (Group 1), 89 as 'probably carcinogenic' (Group 2A), 318 as 'possibly carcinogenic' (Group 2B), and 499 as 'not classifiable as to carcinogenicity' (Group 3). Many Group 1 agents derived their 'sufficient evidence of cancer in humans' from studies of exposed workers. Since 1991, it has been possible to classify an agent in Group 1 based on strong mechanistic evidence in exposed humans and sufficient evidence from cancer bioassays when human cancer evidence was less-than-sufficient. In the recently revised Preamble for the IARC Monographs, mechanistic evidence has gained increased prominence as an individual evidence stream, reflecting advances in mechanistic toxicology and molecular epidemiology. The Preamble revision introduces new possibilities for carcinogen identification from robust mechanistic studies in exposed humans. Specifically, strong evidence that an agent exhibits 'key characteristics' (KCs) of carcinogens in exposed humans can lead explicitly to a Group 2A evaluation when evidence of cancer in humans is limited. Further, classification in Group 2B can be based on strong evidence of KCs in exposed humans alone. Thus, especially for agents for which cancer studies in experimental systems are impracticable (e.g., work as a firefighter), mechanistic studies in exposed humans can increasingly play a crucial role in cancer hazard identification. We will address critical aspects of study design, exposure assessment, and KC-related endpoints anticipated to be influential in future Monographs evaluations of mechanistic studies.
We will draw examples from mechanistic studies in workers that contributed substantively to previous Monographs evaluations, and from ongoing occupational studies of agents accorded high priority for future evaluation by the IARC Monographs (e.g., carbon nanotubes).

\section{S-153 NIGHT SHIFT WORK INTERVENTIONS - WHAT DO WE NEED TO KNOW TO MAKE A DIFFERENCE?}

${ }^{1}$ Anne Helene Garde. 'National Research Centre for the Working Environment, Denmark

\subsection{6/OEM-2021-EPI.416}

In a modern society, night shift work is inevitable in many sectors including healthcare, industry and transport, and it is important to reduce the potential harm by preventing negative effects for health and safety from night shift work.

This presentation is part of the symposium 'Night shift work research: what we need to know to make a difference?'. The aim of the symposium is to present evidence on night work and chronic disease risk and to identify and discuss how to move forward in etiological and prevention research to provide conclusive evidence for action.

Night shift work interventions can be defined as change strategies with the purpose to reduce health and safety risks associated with night shift work. They may be directed towards the organization or towards the individual and may address different possible mechanisms linking night shift work to health and safety risks.

An example of an intervention directed towards the organization is changes in scheduling of night shift work e. g. changes in number of consecutive night shifts; duration of shift intervals; or shift duration, which are all known to be associated with possible mechanisms linking night shift work to disease and accidents. Examples of interventions directed towards the individual are light interventions, which are related to experience of fatigue and diurnal disruption, and sleep hygiene interventions aimed at reducing the negative impact of night shift work on sleep duration and quality.

This presentation contributes to the following panel discussion on what do we need to know in order to implement such prevention measures by presenting relevant examples of possible interventions. Particular focus will be on what is known about success and barriers for implementation of interventions based on our own and others research.

\section{S-176 SOCCER 2.0 AND SOCCER IN THE FIELD: MOVING FROM CODING OCCUPATION AFTER DATA COLLECTION TO CODING IN REAL TIME BY STUDY SUBJECTS}

${ }^{1}$ Melissa Friesen, Daniel Russ, Catherine Lerro, Pabitra Josse, Gabriela Andreotti, Debra Silverman, Laura Beane Freeman. ' U.S. National Cancer Institute, United States

\subsection{6/OEM-2021-EPI.417}

Objective Free-text job descriptions from lifetime occupational history questionnaires are the starting point for nearly all occupational exposure assessment activities in epidemiologic studies. This information is used to code job descriptions into standardized occupation classification (SOC) systems. We describe updates to SOCcer, an algorithm that incorporates 
natural language processing to automatically code job descriptions to SOC-2010.

Methods We recently released SOCcer 2.0. It improved on the original algorithm by 1 ) expanding the training data set to include job descriptions from population-based epidemiologic studies and 2) revising the scoring algorithm to account for nonlinearity in the classifiers. However, perfect prediction is not possible because of the lack of gold standard approach on which to train the algorithm and the brevity of the job descriptions provided by participants, which may fit multiple codes. We have adapted SOCcer to be used in the data collection process to allow the study participant to serve as their own coder when completing a web-based occupational questionnaire. SOCcer reads the participants open-ended job title and tasks responses in real time and proposes a short list of best-fitting SOC-2010 codes for each job. The study participant reviews the list and selects the code that best fits their job.

Results In a validation set of 11,943 jobs, SOCcer' highest scoring code had $50 \%$ and $63 \%$ agreement with a consensus expert-assigned code at the 6- and 3-digit level, respectively. Agreement increased linearly with algorithm score. The expert's code was in the top 3 scoring codes from SOCcer for $>70 \%$ of the jobs, lending support for providing a short list of codes for the study participants to review. Pilot testing is underway.

Conclusion Automated coding, especially in real time, has the potential to substantially reduce the efforts needed to code jobs in large epidemiologic studies and improve the codes accuracy.

\section{S-186 THE IMPACT OF JOB LOSS ON SELF-INJURY MORTALITY IN A COHORT OF AUTOWORKERS: APPLICATION OF A NOVEL CAUSAL APPROACH}

'Suzanne Dufault, Kevin Chen, Sally Picciotto, Andreas Neophytou, Ellen Eisen. 'University of California, United States

\subsection{6/OEM-2021-EPI.418}

Introduction Recent increases in national rates of suicide and fatal overdose have been linked to a deterioration of economic and social stability. The American auto industry experienced these same pressures beginning in the 1980s with the emergence of a competitive global market.

Objectives Using the United Autoworkers - General Motors cohort as a case study, we examine the impact of employment loss on these self-injury mortality events.

Methods For 29,764 autoworkers employed on or after January 1, 1970, we apply incremental propensity score (IPS) interventions, a novel causal inference approach, to examine how proportional shifts in the odds of leaving active GM employment affect the cumulative incidence of self-injury mortality.

Results Cumulative incidence of self-injury mortality was $0.87 \%$ (258 cases) at the observed odds of leaving active GM employment $(\delta=1)$ over a 45 -year period. A $10 \%$ decrease in the odds of leaving active GM employment $(\delta=0.9)$ results in an estimated $7 \%$ drop in self-injury mortality (239 cases) while a $10 \%$ increase $(\delta=1.1)$ results in a $19 \%$ increase in self-injury mortality (306 cases). We reject the null hypothesis of no incremental effect over the range of effects considered ( $\delta$ in $[0.75,1.25] ; \mathrm{P}=0.02$ ).
Conclusion These results suggest that leaving active employment increases the risk of death due to suicide or drug overdose.

\section{S-197 OCCUPATIONAL BURNOUT: DEFINITION, MEASURE AND PREDICTORS}

${ }^{1}$ Irina Guseva Canu. 'Unisanté / PMU, Switzerland

\subsection{6/OEM-2021-EPI.419}

Introduction Despite more than half a century of research on occupational burnout (OB), little is known about its prevalence, etiology, treatment, or prevention. The lack of consensus on its definition and measurement has led to the use of various arbitrary definitions and measures of $\mathrm{OB}$.

Objectives Within the Network on the Coordination and Harmonisation of European Occupational Cohorts (OMEGA$\mathrm{NET}$ ), we aimed at 1 -assessing the state of the art on $\mathrm{OB}$ recognition in the 37 OMEGA-NET-participating countries, 2elaborating a harmonized consensual definition of $\mathrm{OB}$ as a health outcome, 3-assessing the psychometric validity of the most used $\mathrm{OB}$ inventories, and 4-identifying the $\mathrm{OB}$ risk and protective factors.

Methods The first study was an on-line survey among occupational health specialists. The harmonized consensual definition resulted from the systematic review and semantic analysis of available OB definitions and a Delphi study. The two other studies were systematic reviews.

Results The survey showed that in 14 (38\%) countries OB is acknowledged as an occupational disease. The results showed a high variability in $\mathrm{OB}$ diagnosis, in assessment of its workrelatedness, and in conditions allowing compensation of patients. The harmonized definition of $\mathrm{OB}$ was formulated in accordance with the Systematized Nomenclature of Medicine Clinical Terms and consensually approved by 50 experts from 29 countries. Among five most used tools for OB measurements, only two have a moderate evidence of their psychometric validity, the CBI and OLBI. Regarding the $261 \mathrm{OB}$ predictors examined, we found a moderate evidence for the harmful effects of Job demands predictors $n=6$ ) and Negative job attitudes and for the protective effect of Adaptive coping (small effect sizes) and Leisure (small to medium effect sizes).

Conclusions These results will help harmonizing the future research, through the use of the harmonized definition and the most valid measurement tools, and facilitate decision-making and interventions regarding $\mathrm{OB}$.

\section{S-207 MULTIDIMENSIONAL EMPLOYMENT QUALITY, RETIREMENT TRAJECTORIES AND CARDIOMETABOLIC HEALTH IN LATER LIFE IN THE US}

'Sarah Andrea, Jerzy Eisenberg-Guyot, Trevor Peckham, Vanessa Oddo, Anjum Hajat. ${ }^{1}$ Rhode Island Hospital, United States and OHSU-PSU School of Public Health, United States

\subsection{6/OEM-2021-EPI.420}

Introduction The working lives of Americans have become less stable over the past several decades and older adults may be particularly vulnerable to these changes in employment quality (EQ).

Objective We examined later-life multidimensional EQ and retirement trajectories of older Americans and the potential 